\title{
Production of W+jets in Relativistic heavy-ion collisions
}

\author{
Shan-Liang Zhang ${ }^{* a}$, Xin-Nian Wang ${ }^{a b}$, Ben-Wei Zhang ${ }^{a}$ \\ ${ }^{a}$ Institute of Particle Physics and Key Laboratory of Quarks and Lepton Physics (MOE), Central \\ China Normal University, Wuhan 430079, China \\ ${ }^{b}$ Nuclear Science Division Mailstop 70R0319, Lawrence Berkeley National Laboratory, \\ Berkeley, CA 94740 \\ E-mail: zhangshanl@mails.ccnu.edu.cn, xnwang@lbl.gov, \\ bwzhang@mail.ccnu.edu.cn
}

\begin{abstract}
We carry out a detailed calculations of $\mathrm{W}+$ jets production in $\mathrm{Pb}+\mathrm{Pb}$ collisions at the Large Hadron Collider (LHC). In our calculations, the production of $\mathrm{W}+\mathrm{jet}$ in $\mathrm{p}+\mathrm{p}$ reference is obtained from Sherpa, which matches next-to-leading-order matrix elements to the resummation of parton shower calculations. Jet propagation and medium response in the quark-gluon plasma is simulated with the Linear Boltzmann Transport (LBT) model. We calculate five observables of $\mathrm{W}+\mathrm{jets}$ productions with jet quenching effect in $\mathrm{Pb}+\mathrm{Pb}$ collisions: event distribution as a function of the vector sum of the lepton and jets $\left|\vec{p}_{T}^{\text {Miss }}\right|$, nuclear effects for tagged jet cross sections $I_{A A}$, azimuthal angle correlations $\Delta \phi_{j W}$, mean value of momentum imbalance $\left\langle x_{j W}\right\rangle$, average number of jets per W boson $R_{j W}$. The nuclear modifications of these 5 observables due to jet quenching in $\mathrm{Pb}+\mathrm{Pb}$ relative to that in $\mathrm{p}+\mathrm{p}$ collisions are discussed.
\end{abstract}

HardProbes 2020

1-6 June 2020

Austin, Texas

\footnotetext{
* Speaker.
} 


\section{Introduction}

Vector gauge boson associated with jet production are golden channel to probe the properties of the quark-gluon plasma (QGP) [1]. Jets energies are reduced due to elastic and inelastic scattering with the hot and dense medium when they propagate through the QGP [2], while vector gauge bosons will not participate in the strong-interactions directly, escaping the QGP unmodified. Therefore, the vector gauge boson transverse momentum closely reflects the initial energy of the associated jet before interacting with the hot-dense medium.

Recently, $\gamma+$ jet correlations [3], Z+jet production [4], and $\mathrm{H}+$ jet processes [5] have already been investigated by several theory models and experimental groups in $\mathrm{Pb}-\mathrm{Pb}$ collisions at $\sqrt{s}=$ $5.02 \mathrm{TeV}$. However, so far the production of $\mathrm{W}+$ jets in heavy-ion collisions has not yet been quantitatively studied. For completeness, we will carry out a detailed calculations of $\mathrm{W}+$ jet production in $\mathrm{Pb}+\mathrm{Pb}$ collisions at the LHC [6]. As in Z+jets [4], next-to-leading-order (NLO) calculations do not take the resummation of soft/collinear radiation into account and has only limited number of finial particles. Besides, leading-order matrix elements (ME) merged on parton showers(PS) already contain some high-order corrections from both real and virtual contributions. It is short of additional hard or wide-angle radiation from high-order matrix element calculations. Therefore, one needs improved reference of gauge-boson associated with jets production in $\mathrm{p}+\mathrm{p}$ collisions to study $\mathrm{W}+$ jets correlations in heavy-ion collisions.

\section{Model setup for $\mathbf{W}+$ jet in heavy-ion collisions}

Reference $\mathrm{W}+\mathrm{jets}$ production in $\mathrm{p}+\mathrm{p}$ collisions is simulated using NLO matrix elements perturbative calculations matched to the resummation of parton showers [7, 8] within the Monte Corlo event generator Sherpa [9] at $\sqrt{s_{N N}}=5.02 \mathrm{TeV}$. The differential cross section of W boson associated with jets production as a function of the jet transverse momentum shows well agreement with experimental data [10] as shown in Fig .1(left). And then, EPPS16 modified npdfs are used to study cold nuclear matter effects(CNM), as shown in Fig .1(right). The $p_{T}^{\text {jet }}$ spectrum of jets tagged by $W^{-}$is significantly suppressed, while the $p_{T}^{\text {jet }}$ spectrum of jets tagged by $W^{+}$is significantly enhanced due to CNM effects. However, the effect of CNM on the total $\mathrm{W}\left(W^{+}+W^{-}\right)+$jets is consistent with unity and negligible. Our results is in accordance with the calculation in [11]. All the physical explanation of the difference of jet spectra for $\mathrm{W}+$ jets between $\mathrm{p}+\mathrm{p}$ and $\mathrm{Pb}+\mathrm{Pb}$ collisions should be the result of jet-medium interactions.

The Linear Boltzmann Transport (LBT) model is then used to simulate the propagation, energy attenuation of, and medium response induced by jet partons in the QGP [12]. LBT is based on a Boltzmann equation [12]:

$p_{a} \cdot \partial f_{a}\left(p_{a}\right)=-\frac{1}{2} \int \sum_{i=b, c, d} \frac{d^{3} p_{i}}{(2 \pi)^{3} 2 E_{i}} \times\left[f_{a} f_{b}-f_{c} f_{d}\right]\left|M_{a b \rightarrow c d}\right|^{2} \times S_{2}(s, t, u)(2 \pi)^{4} \delta^{4}\left(p_{a}+p_{b}-p_{c}-p_{d}\right)$

where $f_{i}$ are phase-space distributions of partons, $S_{2}(s, t, u)$ is a Lorentz-invariant regulation condition. Elastic scattering is introduced by the complete set of $2 \rightarrow 2$ matrix elements $\left|M_{a b \rightarrow c d}\right|$, and the inelastic scattering is described by the higher-twist formalism for induced gluon radiation $[13,14,15]$. 

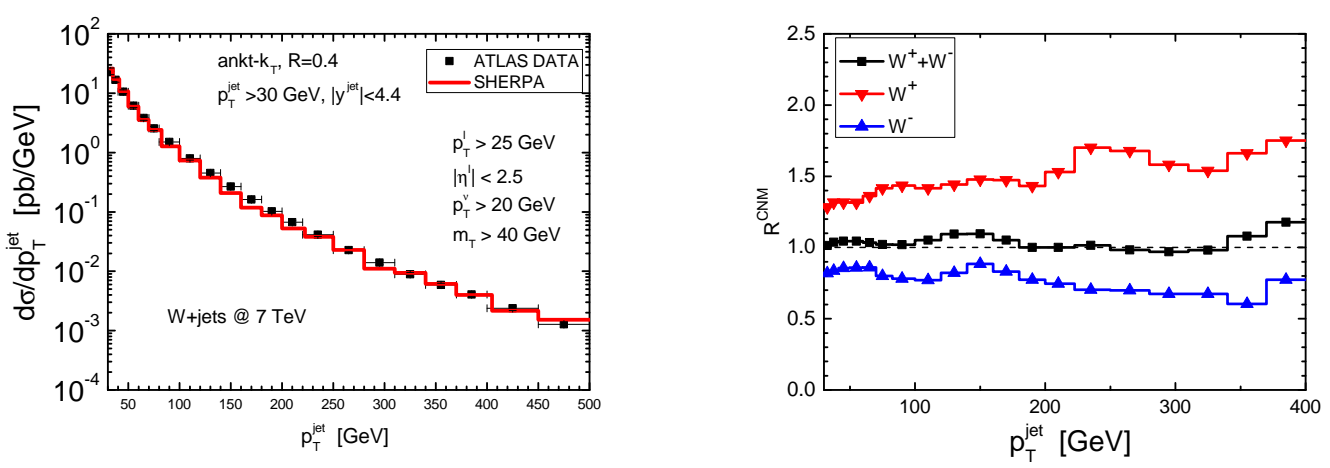

Figure 1: (Color online) Left:Distributions of jet transverse momentum of $\mathrm{W}+$ jets in pp collisions at $7 \mathrm{TeV}$. Right:CNM effects on jet transverse momentum spectra at $\sqrt{s_{N N}}=5.02 \mathrm{TeV}$.

\section{Numerical results}

To be consistent with experimental data, $\mathrm{W}$ and the associated jets are selected according to the kinematic cut adopted by ATLAS experiment [10]. The information of the evolving bulk matter is provided by $(3+1) \mathrm{D}$ hydrodynamics [16].

Firstly, we present an observable defined by the lepton and jets $p_{T}$ as $\vec{p}_{T}^{\text {Miss }}=-\left(\vec{p}_{T}^{l}+\sum \vec{p}_{T}^{\text {jets }}\right)$. Since $\mathrm{W}$ boson eventually decays into an electron and a neutrino which is rather difficult to be measured. So the transverse momentum of neutrino is calculated as the negative vector sum of the $p_{T}$ of electrons, jets, and other soft clusters because of energy-momentum conservation. So, in $\mathrm{p}+\mathrm{p}$ collisions, $p_{T}^{\text {Miss }}$ is just similar equal to the transverse momentum of the neutrino. In $\mathrm{Pb}+\mathrm{Pb}$ collisions, it is the vector sum of the transverse momentum that jets radiate out of jet cone and the neutrino transverse momentum. The distributions of $p_{T}^{\text {Miss }}$ from $\mathrm{pp}$ and $\mathrm{PbPb}$ collisions are shown in Fig .2. The distribution of $\vec{p}_{T}^{\text {Miss }}$ is shifted to smaller value in $\mathrm{Pb}+\mathrm{Pb}$ collisions relative to $\mathrm{p}+\mathrm{p}$ collisions. This displacement is a result of that some amount of jets transverse energy is radiated out of jet cone due to elastic and inelastic scattering with the medium and the direction of the momentum carried by partons which are radiated by jet out of jet cone is in the opposite direction of the neutrino or $\mathrm{W}$ boson. It would simplify the experimental measurements because we just need the jets information which can be easily measured and calculated in $\mathrm{W}+$ jets events.

Fig. 3 (left) plots the nuclear modification factor $I_{A A}=\left(d N^{P b+P b} / d p_{T}^{\text {jet }}\right) /\left(d N^{p+p} / d p_{T}^{\text {jet }}\right)$ of jets tagged by a W within four $p_{T}^{W}$ intervals. An enhancement in $p_{T}^{j e t}<p_{T}^{W, c u t}$ region, and a suppression in $p_{T}^{\text {jet }}>p_{T}^{W, \text { cut }}$ region are observed. We find that $I_{A A}$ is quite sensitive to the kinematic cut due to the steeply falling cross-section in $p_{T}^{W}$ region. $\mathrm{W}+$ jet azimuthal correlations $\Delta \phi_{j W}=\left|\phi_{j e t}-\phi_{W}\right|$ in $\mathrm{p}+\mathrm{p}$ and $\mathrm{Pb}+\mathrm{Pb}$ are shown in Fig. 3 (right). It is moderately suppressed in $\mathrm{Pb}+\mathrm{Pb}$ collisions relative to pp collisions. To have a detailed understanding of the suppression, separated contributions from $\mathrm{W}$ plus only one jet and $\mathrm{W}$ in association with more than one jet in both $\mathrm{p}+\mathrm{p}$ and $\mathrm{Pb}+\mathrm{Pb}$ collisions are also revealed in Fig. 3 . We see that $\mathrm{W}+1$ jet dominates in the large angle region and no significant difference between $\mathrm{p}+\mathrm{p}$ and $\mathrm{Pb}+\mathrm{Pb}$ collisions is observed at such high energy scale. However, $\mathrm{W}+$ multi-jet processes dominates in the small angle region and is considerably suppressed in $\mathrm{Pb}+\mathrm{Pb}$ collisions compared to $\mathrm{pp}$ collisions. Because the initial energies of multi-jet are low and its final energy can easily fall below the thresholds. It is the modification of W+multi-jets 

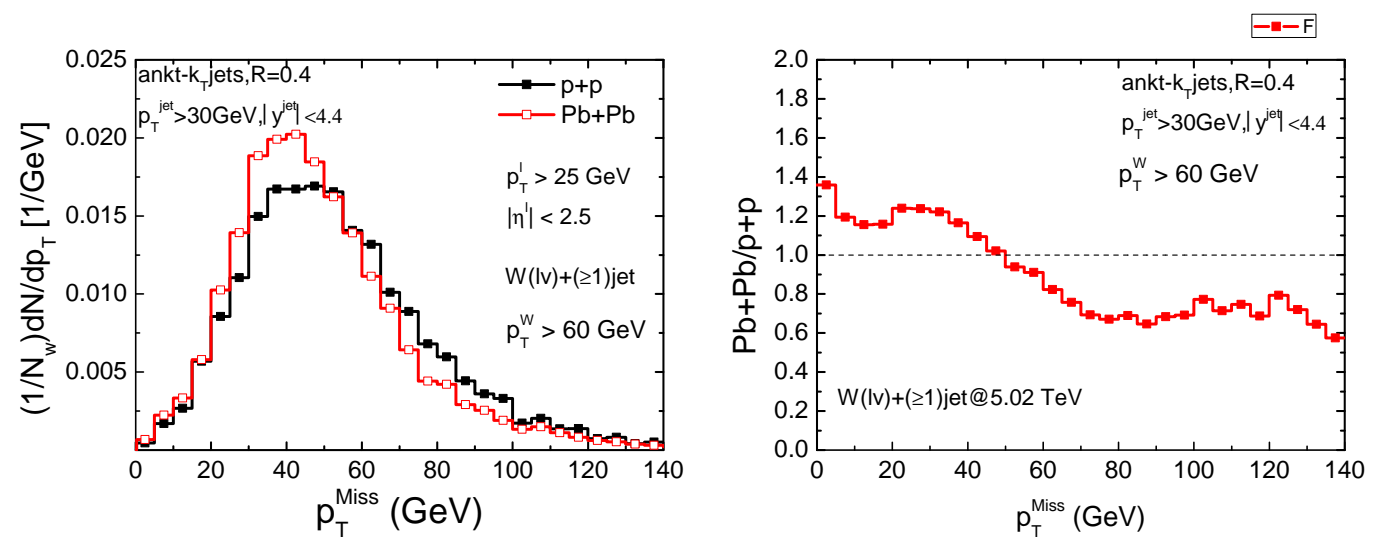

Figure 2: (Color online) Left: Normalized distributions of events passing the $\mathrm{W}+$ jets selection cut as a function of the $\vec{p}_{T}^{M i s s}$. Right: the ratio of distributions in $\mathrm{Pb}+\mathrm{Pb}$ to that in $\mathrm{p}+\mathrm{p}$ collisions at $\sqrt{s}=5.02 \mathrm{TeV}$.
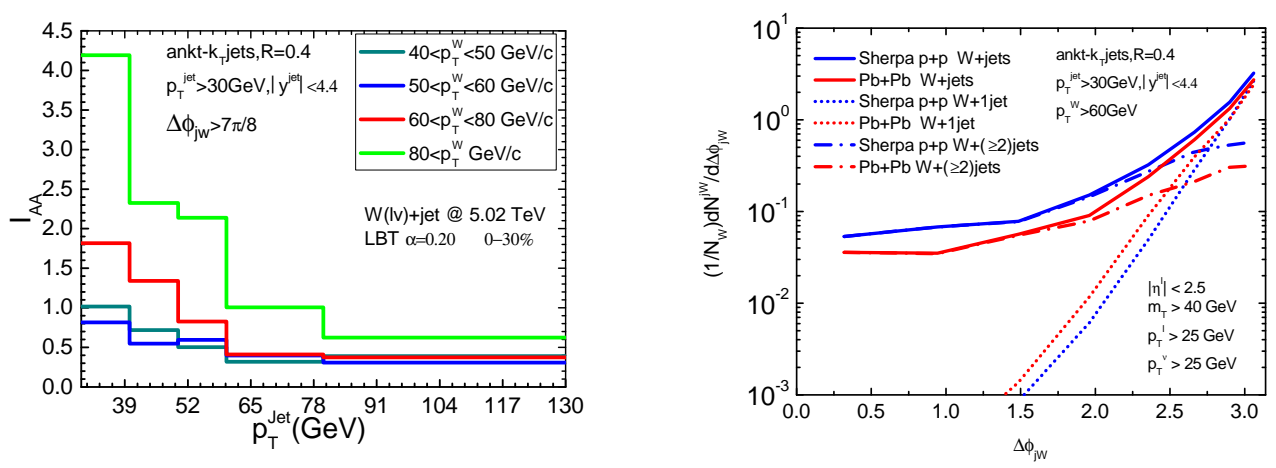

Figure 3: (Color online) Left: nuclear modification factor $I_{A A}$ in $4 p_{T}^{W}$ intervals. Right: azimuthal correlations $\Delta \phi_{j W}$ of $\mathrm{W}+\mathrm{jets}$ in central $\mathrm{Pb}+\mathrm{Pb}$ collisions and $\mathrm{p}+\mathrm{p}$ collisions at $\sqrt{s_{N N}}=5.02 \mathrm{TeV}$.

azimuthal angle difference that leads to the suppression of $\mathrm{W}+\mathrm{jets}$ azimuthal correlations.

And then, the mean value of transverse momentum imbalance between the associated jet and the recoiling $\mathrm{W}$ boson $x_{j W}=p_{T}^{j e t} / p_{T}^{W}$ are presented in Fig. 4 (left). $\left\langle x_{j W}\right\rangle$ in $\mathrm{Pb}+\mathrm{Pb}$ collisions is much smaller than that in $\mathrm{p}+\mathrm{p}$ collisions due to jet energy loss in the medium while the transverse momentum of the $\mathrm{W}$ boson is unattenuated. The difference of the value between $\mathrm{pp}$ and $\mathrm{PbPb}$ collisions increases smoothly as a function of the transverse momentum of $\mathrm{W}$ boson. It indicates that jets tagged by higher energy $\mathrm{W}$ boson lose larger fraction of its energy. Besides, contributions from Multi-jet processes are essential for high energy W bosons. Average number of jet partners per $\mathrm{W}$ boson $R_{j W}$ are shown in Fig. 4 (right). As can be seen, average number of jet partners per $\mathrm{W}$ boson is overall suppressed in $\mathrm{Pb}+\mathrm{Pb}$ collisions compared to that in $\mathrm{p}+\mathrm{p}$ collisions, which is a result of the reduction of jet yields that pass the selection cut after jet quenching in $\mathrm{Pb}+\mathrm{Pb}$ collisions. Besides, higher energy $\mathrm{W}$ boson loss smaller fraction of its jet partners.

This research is supported by NSFC of China with Project Nos. 11435004, by NSFC under Grants Nos. 11935007, 11221504 and 11890714, by DOE under Contract No. DE-AC02- 

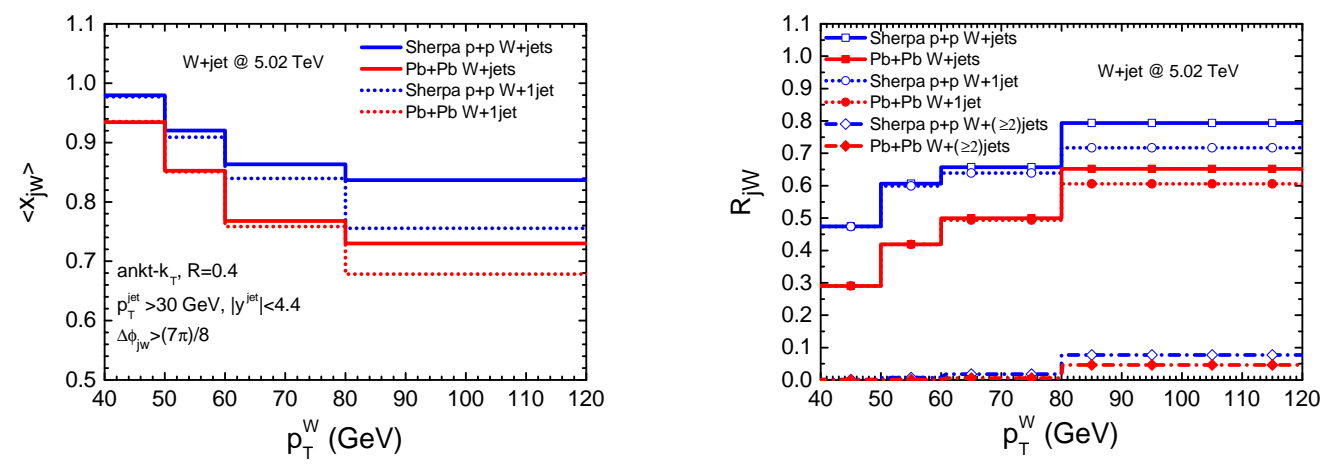

Figure 4: (Color online) (left) Mean value of momentum imbalance, and (right) average number jet partners per $\mathrm{W}$ boson in central $\mathrm{Pb}+\mathrm{Pb}$ collisions and $\mathrm{p}+\mathrm{p}$ collisions at $\sqrt{s_{N N}}=5.02 \mathrm{TeV}$.

05CH11231, and by NSF under Grant No. ACI-1550228.

\section{References}

[1] R. B. Neufeld, I. Vitev and B.-W. Zhang, The Physics of $Z^{0} / \gamma^{*}$-tagged jets at the LHC, Phys. Rev. C 83, 034902 (2011)

[2] M. Gyulassy, I. Vitev, X. N. Wang and B. W. Zhang, Jet quenching and radiative energy loss in dense nuclear matter, In *Hwa, R.C. (ed.) et al.: Quark gluon plasma* 123-191 [nucl-th/0302077].

[3] W. Dai, I. Vitev and B. W. Zhang, Phys. Rev. Lett. 110, no. 14, 142001 (2013).

[4] S. L. Zhang, T. Luo, X. N. Wang and B. W. Zhang, Phys. Rev. C 98, 021901 (2018)

[5] L. Chen, S. Y. Wei and H. Z. Zhang, arXiv:2001.07606 [hep-ph].

[6] S. L. Zhang, X. N. Wang and B. W. Zhang, in preparation.

[7] S. Hoche, F. Krauss, M. Schonherr and F. Siegert, JHEP 1108, 123 (2011)

[8] S. Hoeche, F. Krauss, M. Schonherr and F. Siegert, JHEP 1304, 027 (2013)

[9] T. Gleisberg, S. Hoeche, F. Krauss, M. Schonherr, S. Schumann, F. Siegert and J. Winter, Event generation with SHERPA 1.1, JHEP 0902, 007 (2009)

[10] G. Aad et al. [ATLAS Collaboration], Eur. Phys. J. C 75, no. 2, 82 (2015) doi:10.1140/epjc/s10052-015-3262-7 [arXiv:1409.8639 [hep-ex]].

[11] P. Ru, S. A. Kulagin, R. Petti and B. W. Zhang, Phys. Rev. D 94, no. 11, 113013 (2016).

[12] Y. He, T. Luo, X. N. Wang and Y. Zhu, Phys. Rev. C 91, 054908 (2015)

[13] X. F. Guo and X. N. Wang, Multiple scattering, parton energy loss and modified fragmentation functions in deeply inelastic e A scattering, Phys. Rev. Lett. 85, 3591 (2000)

[14] B. W. Zhang, E. Wang and X. N. Wang, Heavy quark energy loss in nuclear medium, Phys. Rev. Lett. 93, 072301 (2004) [nucl-th/0309040].

[15] A. Schafer, X. N. Wang and B. W. Zhang, Multiple Parton Scattering in Nuclei: Quark-quark Scattering, Nucl. Phys. A 793, 128 (2007)

[16] L. Pang, Q. Wang and X. N. Wang, Effects of initial flow velocity fluctuation in event-by-event $(3+1) D$ hydrodynamics, Phys. Rev. C 86, 024911 (2012) 